\title{
RASMUSSEN'S ENCEPHALITIS: REPORT OF 2 CASES
}

\author{
SAHA NC ${ }^{1}$, YEASIN $M^{2}$, RAHMAN ME ${ }^{3}$, RAHMAN MM ${ }^{4}$, MOLLAH MAH $^{5}$
}

\begin{abstract}
:
Two boys, one was 7 years and the other was 3 years 7 months old, were admitted into Dhaka Medical College Hospital one year apart with almost similar complaints of intractable partial seizure, progressive hemiparesis and cognitive deterioration following an episode of encephalitis. Developmental milestones was age appropriate till occurrence of encephalitis, thereafter started deteriorating with progressive deterioration of cognition, behavior, learning, memory and speech. Different anticonvulsants were tried in optimal doses, yet seizure remained uncontrolled. After admission CSF study, EEG and brain imaging was done. EEG showed unilateral slow waves and MRI revealed unilateral cortical atrophy with hyper intense signals in T2 and FLAIR. According to European consensus statement both cases were diagnosed as Rasmussen's Encephalitis (RE).
\end{abstract}

Key words: Rasmussen's Encephalitis, hemispheric atrophy, seizure.

\section{Introduction:}

Rasmussen's encephalitis (RE) is a very rare but severe immune mediated brain disorder leading to unilateral hemispheric atrophy, associated progressive neurologic dysfunction and partial seizure ${ }^{1}$. This disorder was first described by Rasmussen's and colleagues almost 50 years ago $1,2,3$. No epidemiological data is available regarding its incidence. Only 100 cases have been reported since Rasmussen's original article ${ }^{5}$.It occurs in children under the age of 10 years -rarely at adolescent and adult ${ }^{1,4,8}$.It is characterized by frequent partial seizure leading to Epilepsia Partialis Continua, mental deterioration, speech abnormality, contra-lateral hemiparesis and loss of motor skills ${ }^{1,2,3}$. RE can be divided into two phases, in initial phasemost patients experiences frequent seizure and brain damage over the course of first 8-12 months and then enter into late phase-a phase of permanent but stable neurological deficits ${ }^{1}$. This disease probably have not yet been reported from our country-considering it's rarity and special management plan,two cases of Rasmussen's encephalitis have been reported.

Case-1: A 7 years old boy referred from a medical college to this hospital on 22.06.09 with fever for 4 days along with vomiting, convulsion and unconsciousness of 20 days duration.

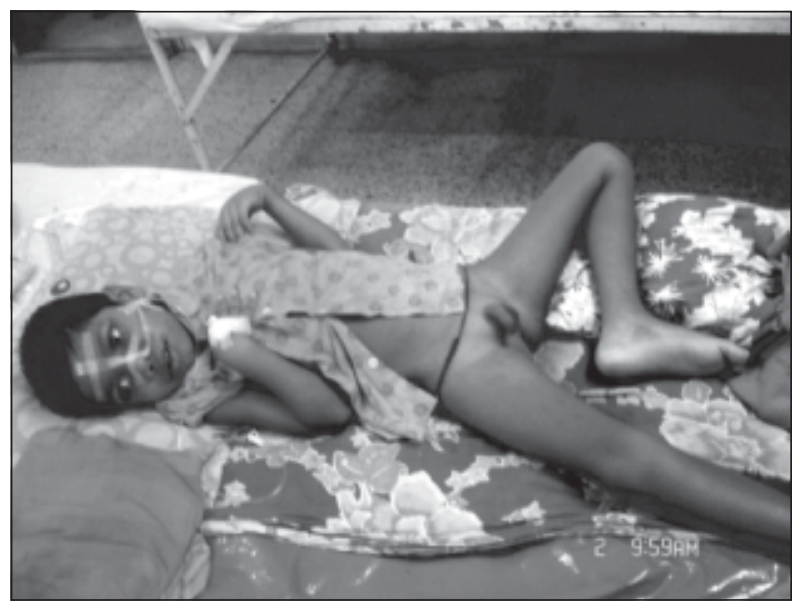

Fig.-1:

1. Associate Professor, Division of Child Neurology, Dhaka Medical College Hospital, Dhaka.

2. Honorary Medical Officer, Department of Paediatrics, Dhaka Medical College Hospital, Dhaka.

3. Professor \& Head, Department of Paediatrics, Dhaka Medical College Hospital, Dhaka.

4. Assistant Professor, Department of Radiology \& Imaging, Dhaka Medical College Hospital, Dhaka.

5. Professor of Neonatology, Dhaka Medical College Hospital, Dhaka.

Correspondence: Dr. Narayan Chandra Saha. 
His birth history was uneventful, completely immunized. Milestone of development was normal till 1 year of age. At that time he had an episode of fever, vomiting, convulsion and unconsciousness. Since then convulsion is persisting as simple partial clonic seizure with progressive weakness and spasticity involving the right side. In spite of different anticonvulsants like Phenobarbitone and phenytoin, seizure frequency was increasing progressively mimicking epilepsia partialis continua. Developmental regression occurred specially motor and cognitive functions. Simultaneously he developed behavioral problem and learning disability for last few years.

On examination at admission GCS was $\mathrm{E} 1+\mathrm{M} 1+\mathrm{V} 1=3$. Sign's of meningeal irritation was present. Also upper motor neuron signs on his right side.

On investigation there was neutrophilic leukocytosis; CSF was hazy, TC was 700/ cumm,neutrophil was 98\%; CSF biochemistry revealed raised protein. EEG showed localized dysfunction with irregular delta waves over fronto temporal region of left hemisphere.CT scan brain(Fig-2) shows gross volume atrophy on left side; MRI also revealed left hemispheric atrophy, cortical and subcortical hyper intensty signal on T2 (Fig-3) and FLAIR(Fig-4) images.

He was diagnosed as a case of Rasmussen's encephalitis with superadded meningitis based

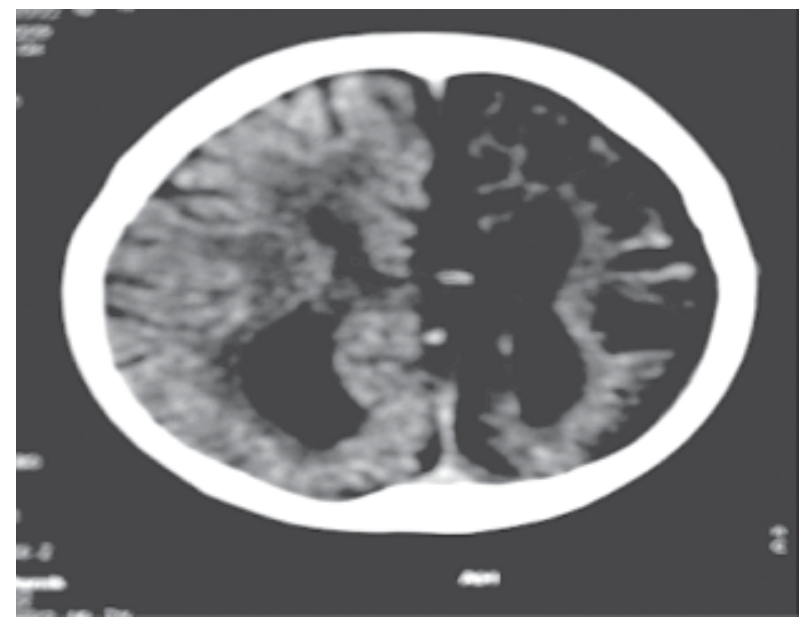

Fig.-2:

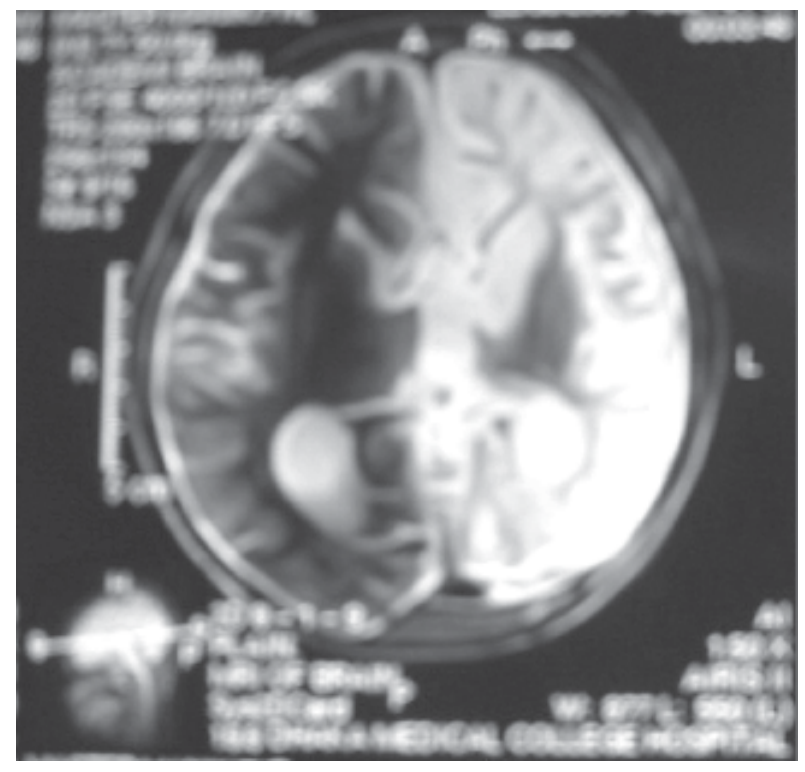

Fig.-3:

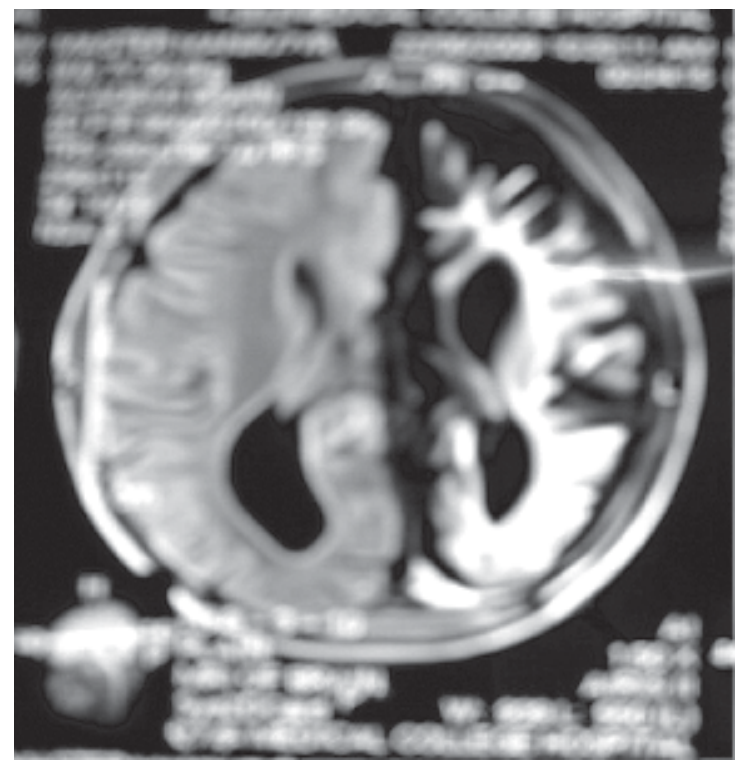

Fig.-4:

on his clinical presentation, investigation profile with particular attention to regression of acquired milestone of development, cognitive and behavioral deterioration, progressive motor weakness and uncontrolled partial seizure inspite of adequate anti epileptic medication. Patient was managed with nutritional support through NG tube feeding, Inj Ceftriaxone IV, control of convulsion was tried with phenytoin and phenobarbitone but without significant improvement. Ultimately he was switched over 
to carbamazepine.He was discharged with improved general condition but with residual disability on the right side with advise of physiotherapy, occupational therapy and come to child neurology clinic to follow up.

At the time of reporting he had high frequency of seizure in spite of optimum doses of both carbamazepine and sodium Valproate.

Case-2: A 3 years 7 months old boy admitted on 09.05.10 with complaints of repeated convulsion and progressive weakness on right side and aphasia for last 7 months.

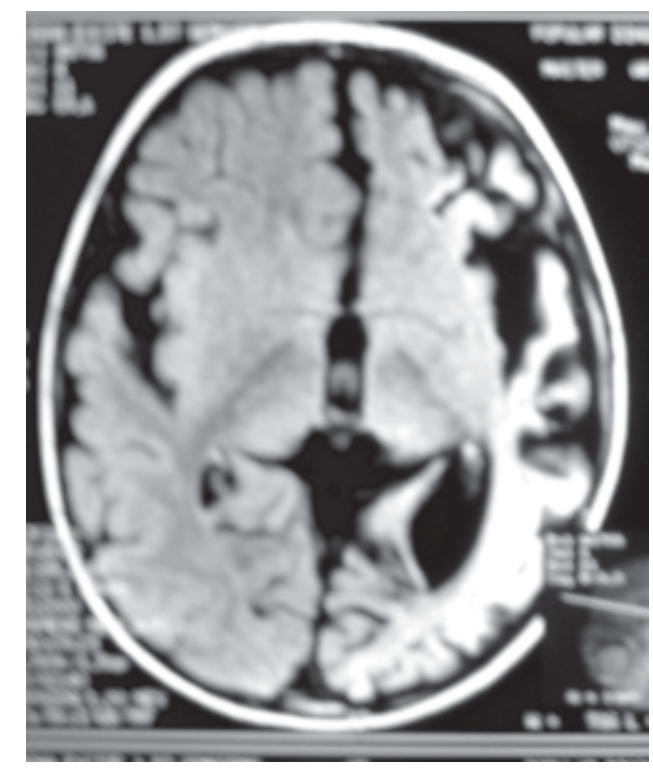

Fig.-5:

His birth history was uneventful and milestone of development was normal till 3 years of age. At that time he had an episode of fever followed by simple partial seizure and unconsciousness. He was treated as a case of encephalitis. After two days of treatment patient regained consciousness but convulsion was persisting as simple partial clonic seizure lasting for a few seconds, 6-8 times a day, for last 7 months. Seizure pattern have been changed to infantile spasm for last few days. Concurrently he developed progressive weakness involving the right side. He received different anticonvulsants like Phenobarbitone, phenytoin, carbamezipine and sodiumvalporate in their optimum doses but seizure was not controlled rather increasing in frequency fulfilling the criteria of Epilepsia partialis continua. Simultaneously he deteriorated in motor and cognitive function, developed progressive aphasia, behavioral problem, partial loss of memory and disorientation for last few months.

On examination patient is conscious but disoriented, aphasic and weakness is present in both upper and lower limbs with muscle power $3 / 5$, and presence of UMN sign on right side.

Regarding investigation, CSF study was normal; EEG demonstrated severe abnormality with frequent high amplitude bilateral epileptiform discharges. CT scan of brain showed volume atrophy on left side and also MRI (fig-05 and fig06) revealed cortical atrophy and encephalomalacic changes on left temporoparieto-occipital region with hyperintense signal on T2 image.

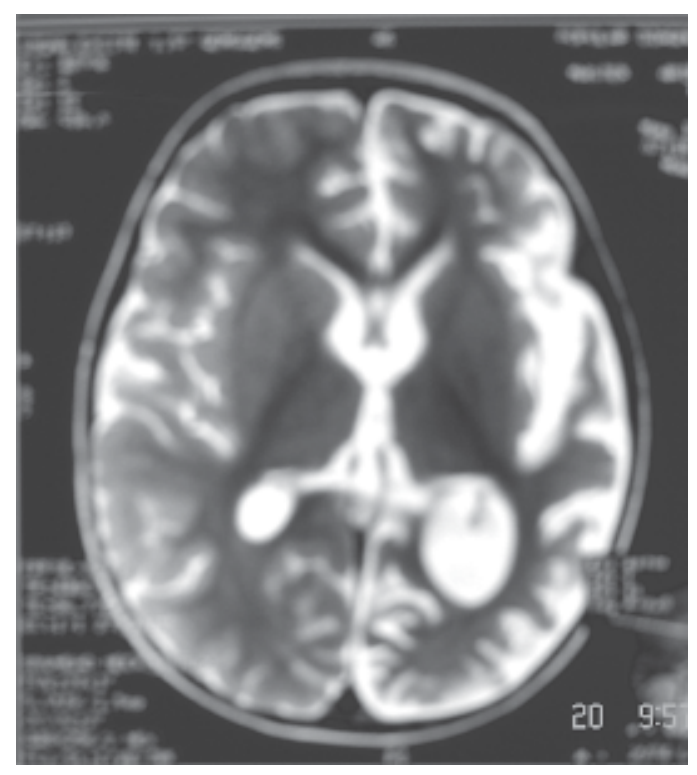

Fig.-6:

Based on classical clinical presentation, deteriorating milestone ,behavioral abnormality and cortical atrophy on neuroimaging the patient was diagnosed as Rasmussen's encephalitis .

The patient was treated with ACTH for infantile Spasm along with supportive measures and finally discharged after reducing seizure 
frequency with improved general condition with advice about physiotherapy, occupational therapy and to attend child neurology clinic for further follow up.

\section{Discussion:}

A recent criteria set by an European consensus statement for diagnosis of Rasmussen's encephalitis is as follows: Part-A :1.Clinical: focal seizure(with or without Epilepsia Partialis Continua) and unilateral cortical deficit 2.EEG: uni-hemispheric slowing with or without epileptiform activity and unilateral seizure onset 3.MRI: Unihemispheric focal cortical atrophy and at least one of the following: Grey or white matter T2 / FLAIR hyper intense signal, Hyper intense signal or atrophy of ipsilateral caudate head. Part-B: Clinical: Epilepsia Partialis Continua or progressive unilateral cortical deficits. MRI: progressive unihemispheric focal cortical atrophy. Histopathology: T cell dominated encephalitis with activated microglial cells and reactive astrogliosis. All 3 criteria should be present for the diagnosis of $\mathrm{RE}$ for part-A and 2 out of 3 for Part- $\mathrm{B}^{1,9}$. Differential diagnosis of RE includes neurometabolic or neurodegenerative diseases, MELAS syndrome, stroke, SSPE, multiple sclerosis, tuberous sclerosis and Sturge-Weber-Syndrome and $\mathrm{HHE}^{1}$. Reported cases fulfill all the criteria as stated above.

Moreover they do not fulfill the criteria of above mentioned differential diagnosis. Both of the cases have intractable seizure and hemiparesis which did not improve or remain static over the course of time .Additionally they had progressively deteriorating symptoms such as cognitive defect, behavioral abnormality, learning disability and aphasia which became evident over the course of time. The age of presentations of the reported cases also similar to the cases elsewhere reported ${ }^{6}$ EEG may contribute to the tentative diagnosis of $\mathrm{RE}$ already in early disease stages. The following uni-hemispheric finding strongly suggest RE in a typical case: impairment of background activity and sleep spindles; focal slow activity; multifocal ictal discharges; subclical ictal discharges ${ }^{1}$.EEG features of case-M having unihemispheric delta wave slowing corroborates with EEG features of RE as stated above. The EEG features of case-A was generalized sharp and slow wave complexes interspersed with burst of spikes and wave of long duration which is similar to the findings of Gloor, who found bilaterally independent ictal onsets in 3 out of 32 patients ${ }^{1}$. The neuroimaging findings of the reported cases has similarity with the MRI features of the European diagnostic criteria.MRI is helpful in diagnosing and monitoring of RE.MRS in severe disease may show increase in lactate and choline peak, decrease in NAA peak, reflecting neuronal and axonal loss, gliosis, and elevated membrane turnover and recent crisis ${ }^{7}$. The treatment modalities of RE includes i.control of seizure which includes antiepilepsy drug therapy, Ketogenic diet, epilepsy surgery (hemispherectomy) in selected cases ii.immunotherapy such as corticosteroids (prednisolone/MPS),IVIG or both can be used in early stage with beneficial effect on control of seizure and better neurological outcome.Tacrolimus has also been tried ${ }^{1}$. As both the case presented at late stage, the aim of treatment was to control of seizure and better rehabilitation through physiotherapy and occupational therapy. It is expected that more cases will be reported in near future with the introduction of recent diagnostic criteria.

\section{Conclusion:}

$\mathrm{RE}$ should be considered as strong differential diagnosis in any intractable partial epilepsy with ipsilateral progressive hemiparesis and cotralateral cerebral atrophy along with declining cognitive and behavioral problem.

\section{Reference:}

1. C.G. Bien, T.Granata,C.Antozzi-Pathogenesis, Diagnosis and treatment of Rasmussen's encephalitis-A European consensus statementBrain 2005, 128,454-471

2. Gordon N: Rasmussen's encephalitis. Dev Med Child Neurol 1996,38:133-136.

3. Antel JP: Rasmussen's encephalitis and the new hat. Neurology 1996,46:9-11.

4. Hart YM, et al.: Chronic encephalitis and epilepsy in adults and adolescents: a variant of Rasmussen's syndrome? Neurology 1997,48:418424. 
5. Andermann E, Oguni H, Guttmann RD, Osterland CK, Antel JP, Eeg-Olofsson O, et al. Genetic aspects of chronic encephalitis. In: Andermann F, editor. Chronic encephalitis and epilepsy. Boston: Butterworth-Heinemann; 1991. p. 16775 .

6. Chipparini L,Granata T,Fariana L,Ciceri E,Erbetta A-Diagnostic imaging in 13 cases of Rasmussen's encephalitis: can early MRI suggest the diagnosis?-Neuroradiology. 2003;45(3); 171-83

7. Faria AV, ReisF, Dabus G.C, Zanardi V.A, Guerreiro M.M, CendesF. MRI findings in the diagnosis and monitoring of Rasmussen's encephalitis; Arq Neuropsiquiatr. 2008; 67(3): 792-7.

8. Hart YM, Andermann F, Fish DR, Dubeau F, Robitaille Y, Rasmussen T, Berkovic S, Marino R, Yakoubian EM, Spillane K, Scaravilli F.. Chronic encephalitis and epilepsy in adults and adolescents: a variant of Rasmussen's syndrome? Neurology 1997;48: 418-24.

9. Bien CG, Urbach H, Deckert Schram J, Wiestler $\mathrm{OD}$, Lassmann $\mathrm{H}$, Elger CE.. Diagnosis and staging of Rasmussen's encephalitis by serial MRI and histopathology. Neurology 2002;58: 250-7. 